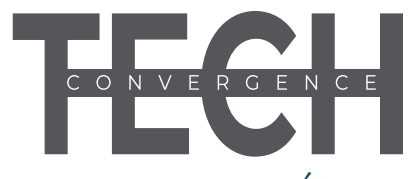

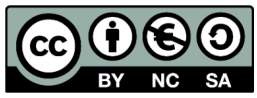

REVISTACIENTIIFICA

Marzo-Agosto 2020

Vol. 4, No. 1,36-48

ISSN 2737-6087

\title{
QUIET BOOK: AULA SENSORIAL DIGITAL
}

\author{
Mariela De Jesús Pauta Yanza ${ }^{1}$, Daniel Claudio Perazzo Logioia ${ }^{2}$ \\ ${ }^{1}$ Tecnólogo en Diseño Gráfico \\ amoresmari26@gmail.com \\ (iD) https://orcid.org/0000-0002-9452-9049
}

${ }^{2}$ Vicerrector del Instituto Superior Tecnológico Particular Sudamericano, Cuenca. vicerectoradoits@sudamericano.edu.ec (iD) https://orcid.org/0000-0003-3089-2225

\author{
Fecha de recepción: \\ 15 de noviembre de 2019 \\ Fecha de aceptación: \\ 8 de enero de 2020
}

\section{RESUMEN}

El libro sensorial, llamado también libro tranquilo, libro silencioso, libro suave y su nombre más conocido Quiet Book, es un libro artesanal que ofrece sensaciones de percepción y oportunidades para desarrollar habilidades intelectuales en los niños. Promueve el aprendizaje, utilizando varias actividades que proporcionan múltiples sensaciones y diversas experiencias en los niños, a través del contacto directo con materiales como fieltro, telas de colores, cuentas de madera, botones, cintas, etc. Esta interacción otorga diversos beneficios en los niños como la estimulación y desarrollo de habilidades, ejercita la motricidad fina y la coordinación, potencia la creatividad y despierta la curiosidad; incentiva el hábito de la lectura desde la primera infancia ya que el contacto del niño con actividades como el reconocimiento de formas y tamaños, los colores básicos y las letras permite que se familiarice con el lenguaje a pesar que aún no sea un lector. De igual manera, es una estrategia lúdica de apoyo al proceso de enseñanza - aprendizaje en el aula como material didáctico que estimula el desarrollo de las relaciones lógico - matemáticas (seriación, secuencia, clasificación, etc.) teniendo en cuenta cada etapa de crecimiento del niño (Suárez, 2017). La gran satisfacción de aplicar este método es que ayuda a la psicomotricidad fina, que se relaciona con la vida diaria, estimula el desarrollo sensorial, estimula la coordinación de manos y creatividad, despierta la curiosidad por experiencias como es el; explorar materiales, formas, números, imaginación y emociones.

Los niños aprenderán actividades cotidianas como; abotonarse sus abrigos, atarse los cordones de los zapatos, abrir, cerrar pinzas, texturas, colores, aplastar, coger etc., es para una edad de 11 meses a 6 años ya que desarrolla conceptos de lógica, lenguaje y conocimiento del medio, esta educación se imparte desde temprana edad por que la enseñanza será significativa funcional. (Libros Sensoriales, método Montessori, 2016).

Palabras Clave: sensorial, percepción, motricidad fina y gruesa

fers sensations of perception and opportunities to develop intellectual skills in children. It promotes learning, using various activities that provide multiple sensations and diverse experiences in children, through direct contact with 
materials such as felt, colored fabrics, wooden beads, buttons, ribbons, etc. This interaction provides various benefits in children such as stimulation and development of skills, exercises fine motor skills and coordination, enhances creativity and awakens curiosity; It encourages the habit of reading from early childhood since the child's contact with activities such as the recognition of shapes and sizes, basic colors and letters allow them to become familiar with the language even though they are not a reader yet. In the same way, it is a playful strategy to support the teaching-learning process in the classroom as didactic material that stimulates the development of logical-mathematical relationships (seriation, sequence, classification, etc.) taking into account each stage of the child's growth (Suárez, 2017). The great satisfaction of applying this method is that it helps fine motor skills, which is related to daily life, stimulates sensory development, stimulates hand coordination and creativity, awakens curiosity for experiences such as: explore materials, shapes, numbers, imagination, and emotions.

Children will learn everyday activities like; buttoning their coats, tying shoelaces, opening, closing clips, textures, colors, squashing, catching etc, it is for an age of 11 months to 6 years as it develops concepts of logic, language and knowledge of the environment, this education is taught from an early age because the teaching will be meaningful and functional. (Sensory Books, Montessori method, 2016).

Keywords: sensory, perception, fine and coarse motor

Forma sugerida de citar: Pauta Yanza, M. J. \& Perazzo Logioia, D. C. (2020). Quiet Book: aula sensorial digital. Convergence Tech Revista Científica. 4(1), 36-48.

\section{INTRODUCCIÓN}

$\mathbf{E}$ n la vida cotidiana de los niños, jóvenes y adultos, el aprendizaje en la educación es fundamental en la formación u orientación práctica y metodológica, ayuda al área motriz, al desarrollo y crecimiento intelectual.

La educación empieza desde temprana edad, enriqueciendo al niño como un gran ser humano en cuanto a valores, cultura e integridad, esta enseñanza se da primeramente en casa con nuestros padres, hermanos y sobrinos luego, al ingresar a una guardería, escuela o colegio.

De acuerdo con las normativas nacionales e internacionales, la educación es un derecho de todo ser humano, fortalece a las sociedades haciéndolas justas, equitativas y productivas, creando fuentes de trabajo, mejor estilo de vida y sobre todo ayuda a fortalecer y recuperar el autoestima de los individuos. La educación en los niños debe ser especializada en su primera etapa en donde desarrollará sus habilidades potenciales y bases de aprendizaje, previniendo posibles retrasos del desarrollo de discapacidades.
En cuanto a discapacidades en los niños, la enseñanza deberá ser garantizada, ya que permitirá el acceso a grandes oportunidades; debido a la educación especial que deben tener y pese a la vulnerabilidad a riesgo del desarrollo, los niños sueles ser rechazados, ignorados hasta golpeados en los programas de desarrollo infantil, esto ha hecho que sus familiares se enfrenten a barreras, a actitudes negativas y falta de ambiente accesible.

Uno de los grandes inconvenientes de los niños con retraso del desarrollo $u$ aprendizaje o discapacidad puede agravarse y provocar resultados negativos permanentes, mayor pobreza, baja autoestima y exclusión social.

En la ciudad de Cuenca, provincia del Azuay existe un $26.81 \%$, de niños con discapacidades intelectuales desde 0 a 6 años de edad según (CONADIS, 2019) y por la falta de dinero de los padres los niños no han asistido a un centro educativo que les permita explotar sus destrezas, habilidades y conocimientos, sobre todo son escasos los centros infantiles que trabaje con este tipo de enseñanza especial.

Mientras tanto, la visión es aplicar un nuevo método en el ámbito educativo infantil de la ciudad de Cuenca 
para trabajar con estos niños que tienen discapacidades intelectuales, creando conciencia, paciencia y nueva enseñanza mediante un libro sensorial exclusivamente para la Unidad Educativa Sudamericano, quienes aplicarán este reto y gran proyecto para su área disciplinaria y enseñanza.

El objetivo de la investigación consiste en diseñar y desarrollar un libro neurosensorial con herramientas que favorecen el aprendizaje de niños y niñas de 1 a 5 años con discapacidad intelectual leve, aplicado al proyecto de investigación del Instituto Tecnológico Particular Sudamericano, de la ciudad de Cuenca, provincia del Azuay.

Dentro de los resultados esperados se estima que los niños aprendan actividades cotidianas como; abotonarse sus abrigos, atarse los cordones de los zapatos, abrir, cerrar pinzas, texturas, colores, aplastar, coger etc., es para una edad de 11 meses a 6 años ya que desarrolla conceptos de lógica, lenguaje y conocimiento del medio, esta educación se imparte desde temprana edad por que la enseñanza será significativa funcional. (Libros Sensoriales, método Montessori, 2016).

\section{DESARROLLO}

De acuerdo con la (UNESCO, 2017) la educación inclusiva expresa un proceso de identificar y responder a la diversidad de las necesidades de todos los estudiantes a través de la mayor participación en el aprendizaje, las culturas y las comunicaciones en función de reducir la exclusión en y desde la educación. La inclusión implica el acceso a una educación de calidad sin ningún tipo de discriminación, lo cual exige una transformación profunda de los sistemas educativos.

Para (Guijarro, 2008) la educación inclusiva se fundamenta en principios éticos, sociales, educativos y económicos. Es un medio para hacer efectivo el derecho a una educación de calidad sin discriminaciones y en igualdad de oportunidades. Constituye un bien común y un derecho humano fundamental del que nadie puede estar excluido, gracias a ella es posible el desarrollo de las personas y de las sociedades.

De tal manera que, el derecho a la educación en su sentido más amplio va más allá del acceso a una educa- ción obligatoria y gratuita. Su pleno ejercicio exige que ésta sea de calidad, promoviendo el máximo desarrollo de las múltiples potencialidades de cada persona, es decir el derecho a la educación es el derecho a aprender a lo largo de la vida.

Otro concepto de (Educación Inclusiva, 2016) sostiene que al hablar de educación inclusiva nos referimos a una aproximación estratégica, diseñada para facilitar el aprendizaje de calidad para los niños, niñas y jóvenes. Son metas comunes trazadas con el propósito de disminuir y superar todo tipo de exclusión desde perspectivas del derecho humano a una educación. Tiene que ver con acceso, participación y aprendizaje exitoso en una educación de calidad para todos. Involucra la defensa de igualdad de oportunidades para todos los niños y niñas, remover todas las barreras para el aprendizaje y facilitar la participación de todos los estudiantes vulnerables a la exclusión y la marginalización.

En este sentido, la Inclusión no es una acción sino un proceso. Es una búsqueda constante para responder más y mejor a la diversidad en términos dinámicos, propios de un camino tortuoso nada fácil. Identifica, elimina barreras, busca la presencia, la participación y éxito de todos los alumnos se basa a la calidad de experiencias en cuanto a resultados de aprendizaje, incorpora la voz del alumnado, sus opiniones, haciéndoles partícipes en las rutinas educativas.

La inclusión vela por los grupos de riesgo. Se incluye los sujetos provenientes de la marginación social, exclusión o bajo rendimiento etc. (Miles, 2019)

Por su parte, UNESCO aprobó la Convención relativa a la Lucha contra las Discriminaciones en la Esfera de la Enseñanza CDEE, tipificando tal figura como "toda distinción, exclusión, limitación o preferencia, fundada en la raza, el color, el sexo, el idioma, la religión, las opiniones políticas o de cualquier otra índole, el origen nacional o social, la posición económica o el nacimiento, que tenga por finalidad o por efecto destruir o alterar la igualdad de trato en la esfera de la enseñanza"

No obstante, es evidente que la sociedad se ha encargado de excluir a muchos niños, jóvenes y adultos de la vida cotidiana que por una o varias condiciones de discapacidad no puede hacer sus labores normalmente, esto 
ha hecho que sean vulnerables, sean inseguros y tengan miedo de explorar otras culturas, caminos, expresiones etc.

Para efectos del cumplimiento de las disposiciones de la ley y el reglamento, se considerará persona con discapacidad a toda persona que, como consecuencia de una o más deficiencias físicas, mentales y/o sensoriales, congénitas o adquiridas, previsiblemente de carácter permanente se ve restringida en al menos un treinta por ciento de su capacidad para realizar una actividad dentro del margen que se considera normal, en el desempeño de sus funciones o actividades habituales (CONADIS).

La Clasificación internacional del funcionamiento de la discapacidad y de la salud, (versión niños y jóvenes) considera a la discapacidad no como una cuestión puramente biológica ni puramente social, sino más bien como una interacción entre problemas de salud y factores ambientales y personales. La discapacidad puede ocurrir en tres niveles:

- Una deficiencia en la función o la estructura corporal, como las cataratas, que impiden el pasaje de la luz y la sensación de la forma y el tamaño de los estímulos visuales;

- Una limitación en la actividad, como la incapacidad de leer o de moverse por sí mismo;

- Una restricción de la participación, como la exclusión de la escuela. (Unicef , 2013)

Se considera persona con discapacidad a toda aquella que, como consecuencia de una o más deficiencias físicas, mentales, intelectuales o sensoriales, con independencia de la causa que la hubiera originado, ve restringida permanentemente su capacidad biológica, psicológica y asociativa para ejercer una o más actividades esenciales de la vida diaria (Ley Orgánica de Discapacidades, 2012).

La Asociación Americana de Discapacidades Intelectuales y del Desarrollo, modificó el término Retraso Mental por Discapacidad Intelectual que se caracteriza por limitaciones significativas tanto en el área intelectual y conducta adaptativa de tipo social y práctico.

- Área Intelectual. Llamado también como inteligencia, se refiere a la capacidad mental general, como el aprendizaje, razonamiento, resolución de problemas etc.

- Conducta Adaptativa. Conjunto de habilidades conceptuales sociales y prácticas que las personas aprenden en su vida diaria.

- Habilidades Conceptuales. Lenguaje y Alfabetización, concepto de dinero, matemático, tiempo.

- Habilidades Sociales. Habilidades interpersonales, responsabilidad social, autoestima, credibilidad, capacidad de seguir reglas, y evitar ser víctimas.

- Habilidades Prácticas. Actividades de la vida diaria, habilidades ocupacionales, uso del teléfono, uso del dinero etc. (Asociación Americana de Discapacidades Intelectuales y del Proceso , 2019)

En cuanto a la Discapacidad Intelectual (López, 2019), indica que se caracteriza por las limitaciones significativas para el buen funcionamiento en la vida diaria, así como para la comprensión e interacción con el entorno. No se considera una enfermedad mental. Existen diferentes tipos de apoyo según la autonomía de las personas:

- Intermitente. Necesidad de apoyo esporádico.

- Limitado. Necesidad de apoyo para ciertas tareas.

- Importante. Necesidad de apoyo diario.

- Profundo. Necesidad de apoyo para todas las tareas diarias, incluyendo cuidados especializados y continuos.

De igual manera, (López, 2019) sostiene que es habitual en las personas con discapacidad Intelectual que muestren un lenguaje equivalente al de una persona de menor de edad, cuanto más grave sea la discapacidad intelectual mayores problemas aparecerán. Puede haber alteraciones de conducta, problemas emocionales, asociados a la exclusión y discriminación, a la frustración por no poder expresar sus necesidades y deseos por el mismo hecho de no poder transmitir bien un mensaje.

Las causas de la discapacidad intelectual se puede decir que existe cuatro tipos de riesgo: biomédico, conductual, educativo y social. 
- Causas Prenatales, antes del parto.

- Causas perinatales, durante el parto.

- Causas posnatales, tras el parto.

Los diferentes tipos de discapacidad intelectual están clasificados por niveles de deficiencia cognitiva, no por síndrome, pero algunos de estos síndromes se clasifican entre algunos de los niveles de deficiencia:

- Discapacidad intelectual leve. Se caracteriza porque quienes la tienen se sitúan entre $50 \%$ a $70 \%$ en cociente intelectual y el $85 \%$ se sitúan en ese nivel. La mayoría de las personas con este tipo de discapacidad son capaces de ser integrados en el medio de educación, laboral incluso tener una vida cotidiana sin grandes problemas; son capaces de tener una conversación larga pero medio lenta.

- Discapacidad intelectual moderada. Se caracteriza por estar debajo de $50 \%$ en cociente intelectual, conlleva la necesidad constante de una supervisión, tanto en la educación como en el trabajo, aunque con mucha terapia pueden tener cierto grado de autonomía. En este caso, la persona necesita una supervisión ya más seguida, son capaces de ser integrados en el medio educativo pero con tutor, tanto en el trabajo necesitan supervisión en cada momento, pueden tener una conversación bastante complicada en la definición de palabras.

- Discapacidad intelectual grave. Es cuando el cociente intelectual se encuentra entre el 20 y 35\%, haciendo que quienes la padecen necesiten de una supervisión muy continúa, en vista que presentan daños a nivel neurológico. Esto hace que el individuo tenga habilidades reducidas, poca o nula comprensión lectora y numérica. Aquí las personas se comunican con Holo frases y se consideran incapaces de tomar sus propias decisiones.

- Discapacidad intelectual profundo. Es uno de los más infrecuentes es el más temido por los padres, pues quienes la padecen tienen una capacidad de cociente intelectual menos a $20 \%$. Esto implica un cuidado de forma permanente y la tasa de super- vivencia en muy baja, suele acompañarse de problemas neurológicos. Sus habilidades motoras son limitadas y su capacidad comunicativa es bastante baja o inexistente.

- Algunos niños nacen con una deficiencia o un problema de salud discapacitante, mientras que otros pueden padecer discapacidad como resultado de enfermedades, lesiones o mala nutrición. Los niños con discapacidad incluyen los que tienen afecciones de salud como parálisis cerebral, espina bífida, distrofia muscular, lesión traumática de la médula espinal, síndrome de Down, y niños con deficiencias auditivas, visuales, físicas, comunicacionales e intelectuales. Algunos de estos niños tienen una única deficiencia, mientras que otros tienen varias. Por ejemplo, un niño con parálisis cerebral puede tener deficiencias de movilidad, de comunicación e intelectuales. (Unicef , 2013).

En algunos casos, la discapacidad pasa por alto, debemos entender que hay muchos factores donde el niño se hace acreedor a una discapacidad, puede ser por enfermedades desde el vientre o algún accidente ocurrido en la infancia, eso no quiere decir que debemos olvidar la prioridad que necesitan en la educación, en salud, en lo social, porque al incluirlos en cualquier ámbito les ayudaría a perder las inseguridades.

\section{La discapacidad en niños de entre 0 a 6 años de la Ciudad de Cuenca}

En la ciudad de Cuenca se registra 373 niños con diferentes tipos de discapacidad, entre la edad de 0 a 6 años: el $39.68 \%$ son niños con discapacidad física, el $26.81 \%$ tienen discapacidad intelectual, el $10.19 \%$ son niños con discapacidad auditiva, el 6.17\% son niños con discapacidad visual y el $17.16 \%$ tienen discapacidad psicosocial. (CONADIS, 2019). Por lo tanto es un porcentaje significativo, los niños que tienen discapacidad intelectual y por ello la educación debe ser especializada y prioritaria, donde se les otorgue un trato igualitario.

\section{La educación en los niños con discapacidad}

El desarrollo infantil es un proceso dinámico por el cual los niños progresan desde un estado de dependen- 
cia de todos sus cuidadores en todas sus áreas de funcionamiento, durante la lactancia, hacia una creciente independencia en la segunda infancia (edad escolar), la adolescencia y la adultez. En ese proceso adquieren habilidades en varios ámbitos relacionados: sensorial-motor, cognitivo, comunicacional y socio-emocional. El desarrollo en cada ámbito está marcado por una serie de hitos o pasos y típicamente implica el dominio de habilidades sencillas antes de que se puedan aprender habilidades más complejas. Cabe reconocer que los niños pueden tener un papel activo en el desarrollo de sus propias habilidades y que su desarrollo también se ve influido por la interacción con el ambiente (Unicef , 2013).

En concordancia con ello, (Bassedas, 2010) indica que cuando se habla de alumnado con discapacidad intelectual y del desarrollo se refiere a un amplio abanico de alumnos que por razones diversas (neurológicas, cromosomáticas, debido a accidentes, carencia de estimulación) tienen limitaciones en su funcionamiento intelectual.

\section{La importancia de los libros sensoriales para los niños con discapacidad intelectual}

La educación sensorial es muy importante dentro de la vida de toda persona, el educador es la línea principal de intervención para facilitar a los niños un ambiente rico en estímulos, donde se pueda experimentar y explorar como base de la construcción de su desarrollo global, la experimentación como la exploración se van a producir a través de los sistemas sensoperceptivos ayudándonos de esta manera en el desarrollo global de la persona (Elena Antoraz, 2010).

El libro sensorial promueve el aprendizaje utilizando varias actividades que proporcionan múltiples sensaciones y diversas experiencias en los niños a través del contacto directo con materiales como fieltro, telas de colores, cuentas de madera, botones, cintas etc.

Esta interacción otorga diversos beneficios en los niños como la estimulación y desarrollo de habilidades, ejercita la motricidad fina y la coordinación, potencia la creatividad y despierta la curiosidad; incentiva el hábito de la lectura desde la primera infancia ya que el contacto del niño con actividades como el reconocimiento de formas y tamaños, los colores básicos y las letras permite que se familiarice con el lenguaje a pesar que aún no sea un lector, también es una estrategia lúdica de apoyo al proceso de enseñanza - aprendizaje en el aula como material didáctico que estimula el desarrollo de las relaciones lógico - matemáticas (seriación, secuencia, clasificación, etc.) teniendo en cuenta cada etapa de crecimiento del niño (Suárez, 2017).

Por su parte, (Dehasse 1994), indica que el aprendizaje por medio de sensaciones es muy importante porque el ser humano necesita de estímulos para desarrollar sus habilidades, el cerebro es el que va activar la parte sensorial a través del medio ambiente desde su nacimiento. El aprendizaje del niño comienza a partir de la exploración por medio de los sentidos, ya que puede interpretar imágenes; retiene sonidos, figuras, sensaciones.

La información que el niño recibe por medio de los estímulos es sumamente esencial para el comienzo del desarrollo de procesos mentales, ya que el desarrollo de sus habilidades depende de los estímulos sensoriales. El desarrollo sensorial es la base para desarrollar procesos cognitivos; música, sabores, texturas, colores. El niño percibe el estímulo mediante sus sentidos, lo recibe, lo interpreta y esto produce una conducta y esto desemboca en una acumulación de conocimientos para el desarrollo de nuevas tareas.

De tal manera que, el libro sensorial despierta las neuronas del cerebro, haciendo que el niño reaccione a texturas, colores, formas, luces y las pueda reconocer, claro con un aprendizaje constante y resultados lentos.

\section{PROPUESTA DEL QUIET BOOK: AULA SENSORIAL DIGITAL}

Presentamos a continuación, la propuesta de diseño y desarrollo de un libro neurosensorial con herramientas que favorecen el aprendizaje de niños y niñas de 1 a 5 años con discapacidad intelectual leve, aplicado al proyecto de investigación del Instituto Tecnológico Particular Sudamericano.

\section{Introducción}

La Educación Sensorial es muy importante dentro de la vida humana, el Educador es el eje principal de intervención para facilitar a los niños un ambiente sustancioso en estímulos, en donde se pueda experimentar y explorar como base de la construcción de su desarrollo global. 
La experimentación como la exploración se va a producir a través de los sistemas "Senso-Perceptivos", ayudándonos de esta forma en el desarrollo global de la persona.

El libro sensorial promueve el aprendizaje utilizando varias actividades que proporcionan múltiples sensaciones y diversas experiencias en los niños a través del contacto directo con materiales como: fieltro, telas de colores, cuentas de madera, botones, cintas, etc.

Esta interacción tiene como finalidad otorgar diversos beneficios en los niños, como la estimulación, desarrollo de habilidades, ejercitar la motricidad fina y coordinación, que estas a su vez potencian la creatividad despertando la curiosidad e incentivando el hábito de la lectura desde la primera infancia. El contacto del niño con actividades como el reconocimiento de formas, tamaños, los colores básicos y las letras permite que se familiarice con el lenguaje a pesar que aún no sea un lector, también es una estrategia lúdica de apoyo al proceso de enseñanza al aprendizaje en el aula como material didáctico que estimula el desarrollo de las relaciones lógico matemática (seriación, secuencia, clasificación, etc.) teniendo en cuenta cada etapa de crecimiento del niño.

\section{Objetivo}

Aceptar a los niños con discapacidad intelectual leve donde este libro "Neurosensorial" juega un papel importante para mejorar el aprendizaje por medio de actividades lúdicas, cotidianas, básicas e importantes que beneficiará a los movimientos pequeños, a la coordinación, al equilibrio, reconocimiento de formas, texturas y colores, etc.

Esto beneficiará no solamente a un porcentaje de niños, sino a una población entera, de esta forma si un niño mejora su aprendizaje mejora su estabilidad emocional y social, esto favorece a toda una familia.

\section{Materiales}

El libro sensorial está realizado de manera 100\% manual, con fieltro de gramaje $2 \mathrm{~mm}$, fómix, pegamento UHU, imanes de neodimio.

\section{Fieltro}

Es un tipo de tela exclusivamente para hacer manualidades y fue el primer textil creado por el hombre, es un material que no está elaborado con técnica del tejido, se puede moldear, se puede teñir con colorantes y fácil de manipular, su gramaje es de $1 \mathrm{~mm}$ a $3 \mathrm{~mm}$; es muy fácil de cortar, pegar y coser.

\section{Figura $1 »$ Fieltro}

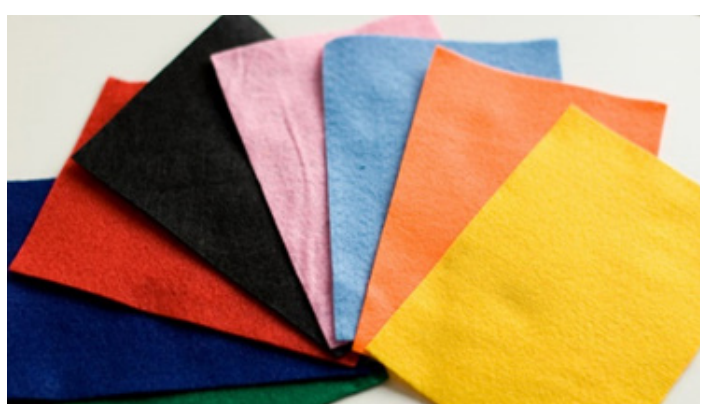

\section{Fomix}

Es un polímero "algo formado por diversos componentes", tipo termoplástico "deformable o flexible" sirve para manualidades, trabajos escolares, terapias, son láminas de diferentes colores y texturas brillantes, rígidas, texturas de algodón, tienen diferentes formas troqueladas que tienen adhesivo (Rodríguez, 2012).

Figura 2 » Fomix

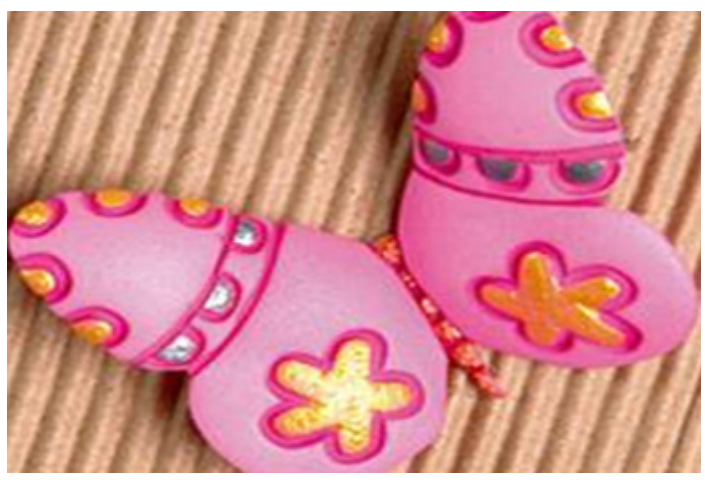

\section{Aerografía}

Instrumento que opera con aire comprimido y sirve para pulverizar pinturas y colores en forma líquida. La boquilla se compone de una válvula de aguja para el líquido y de otra para el aire; se utiliza para el retoque fotográfico, artes decorativas y la pintura. 
Figura 3 » Aerografía

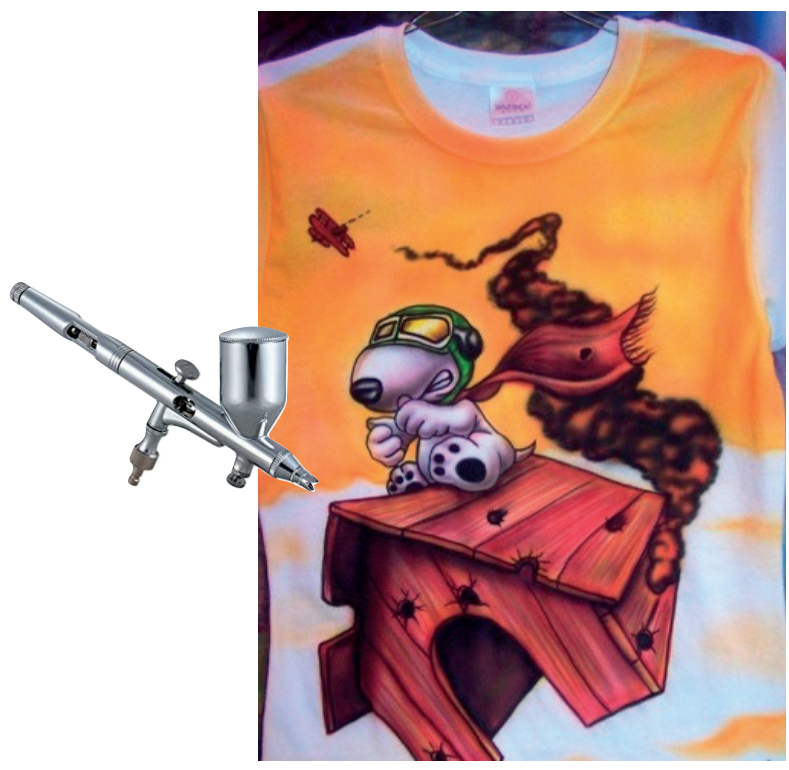

\section{Imán de neodimio}

El neodimio cuando se combina con el boro e hierro forma un imán. Es debido a su apariencia atractiva gracias al níquel, que suelen utilizarse para fines artísticos, pero su utilidad va mucho más allá de ser un simple objeto decorativo.

Figura 4 » Imán

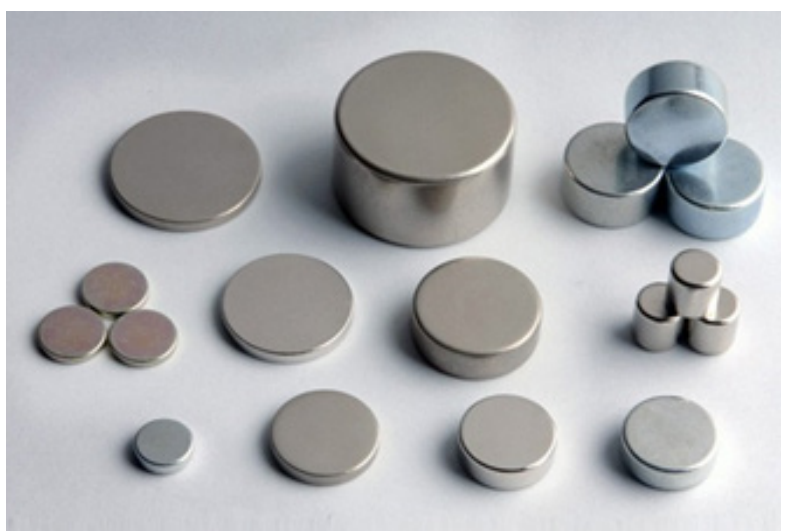

\section{Pegamento textil UHU}

Es un pegamento especial para algodón, tejidos, fieltro, es resistente al lavado hasta 90 grados centígrados, utilizado para manualidades por su grado de resistencia en el pegado.
Figura 5 » Pegamento UHU

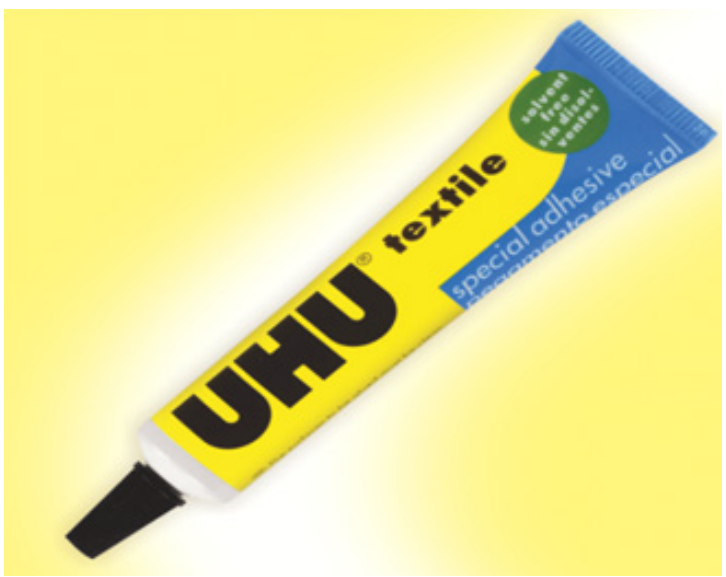

\section{Nano Arduino}

Nano Arduino es una placa de desarrollo de tamaño compacto, completa, basada en el microcontroladores. Tiene 14 pines de entrada/salida digital conexión MiniUSB, terminales para conexión ICSP y un botón de reseteo.

Figura 6 » Nano Arduino

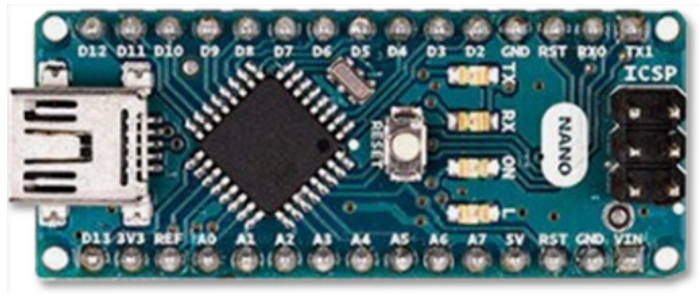

\section{Luces Led}

Los sistemas LED producen luz basándose en un efecto llamado electroluminiscencia, emiten fotones que determinan el color de la luz. El color será diferente dependiendo de los materiales que compongan las cápsulas de los diodos de su interior. (Penélope, 2015)

Figura 7 » Luces Led

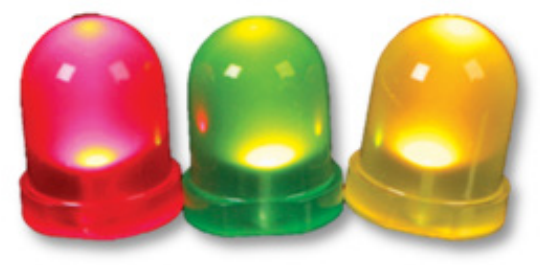




\section{Pulsantes}

Son pequeños pulsadores, son bastante económicos y se pueden 'pinchar' directamente en una placa de prototipado. Estos dispositivos mecánicos tienen 4 patillas.

Figura 8 » Pulsante

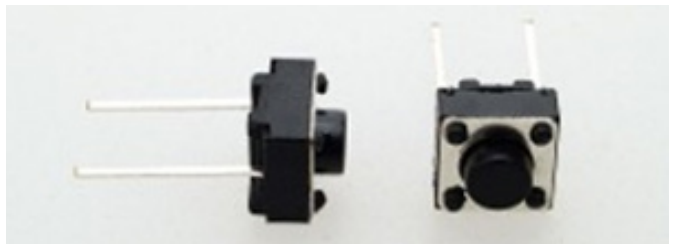

\section{Interruptor}

Los interruptores eléctricos, son dispositivos que sirve para apagar o encender un foco. (E. Parra, 2013)

Figura 9 » Interruptor

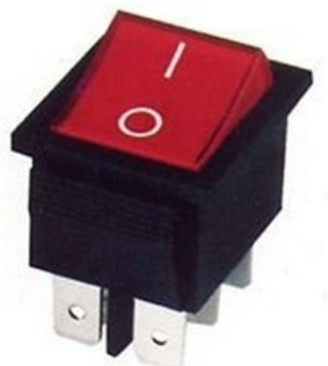

\section{Propuesta del libro "Desarrollo"}

\section{Revisión de homólogos}

El análisis de homólogos nos sirve para tener más clara una idea, en cuanto a libros interactivos y sensoriales se puede obtener mejores resultados en la creación.

Figura 10 » Dibujo_parte1

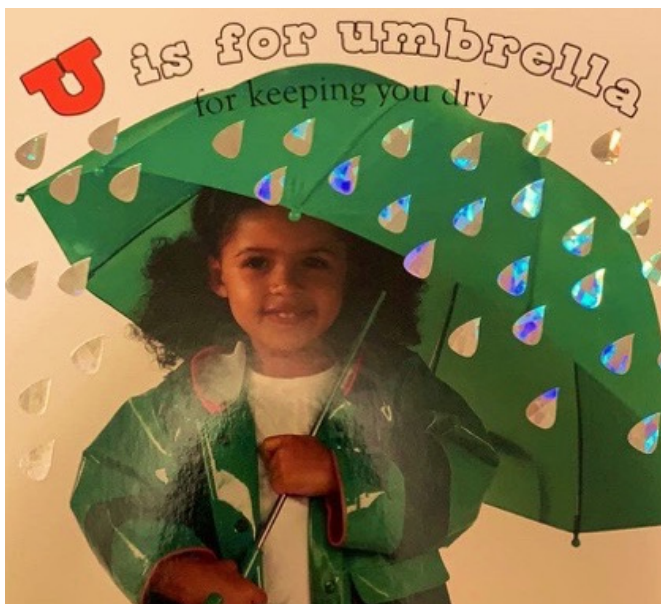

Esta textura del libro en las lluvias, está hecho de lentejuelas, causa un efecto, donde el cerebro empieza a receptar y el niño obtendrá una sensación al tocarlo.

\section{Figura 11 » Dibujo_parte2}

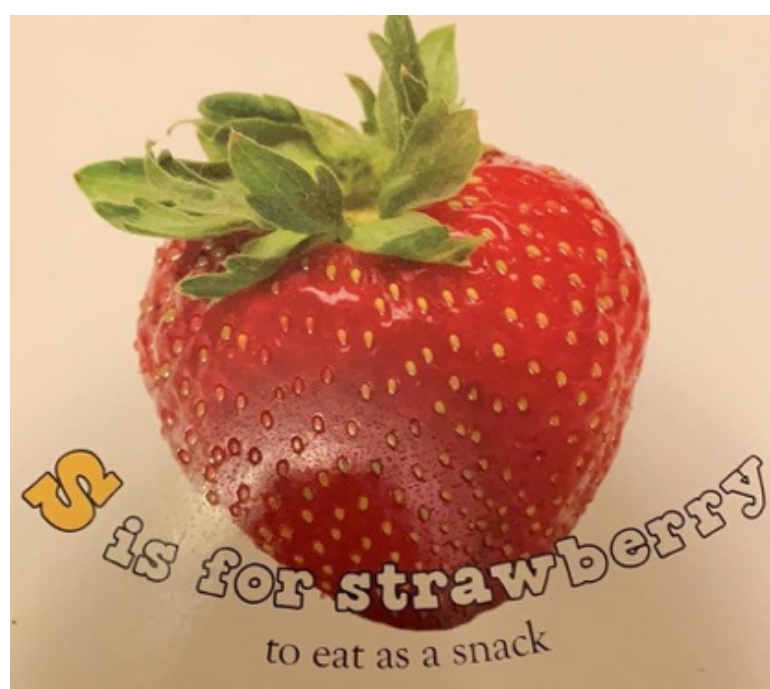

En cuanto a la frutilla, la textura que tiene, son pepas de ajonjolí, sirve para el sentido del tacto.

Figura 12 » Dibujo_parte3

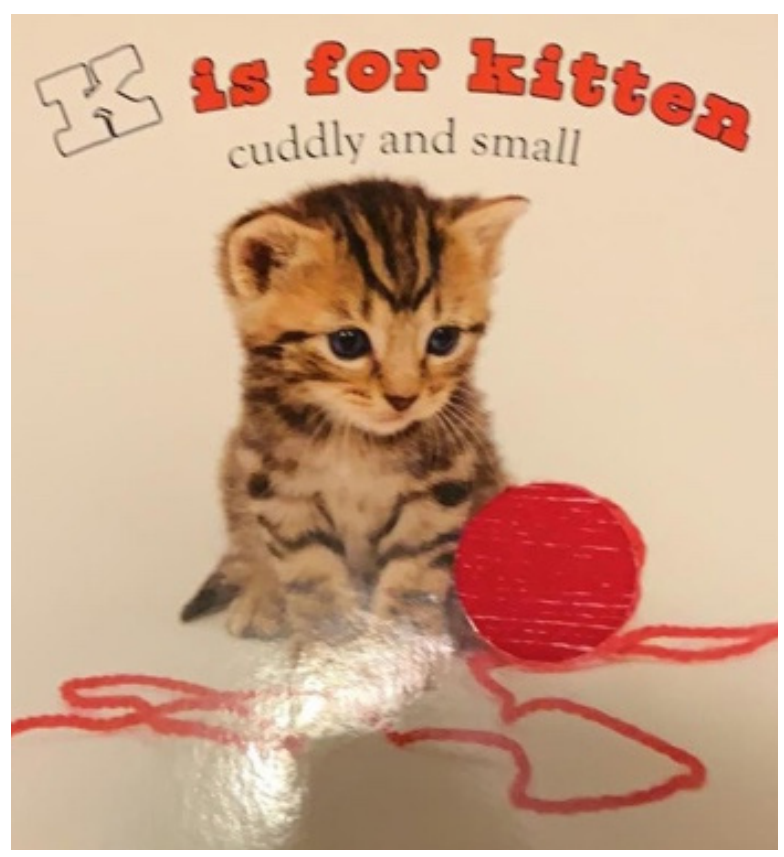

En cuanto al gato, la textura del círculo rojo es propiamente hilo, para el sentido del tacto. 
Figura 13 » Dibujo_parte4
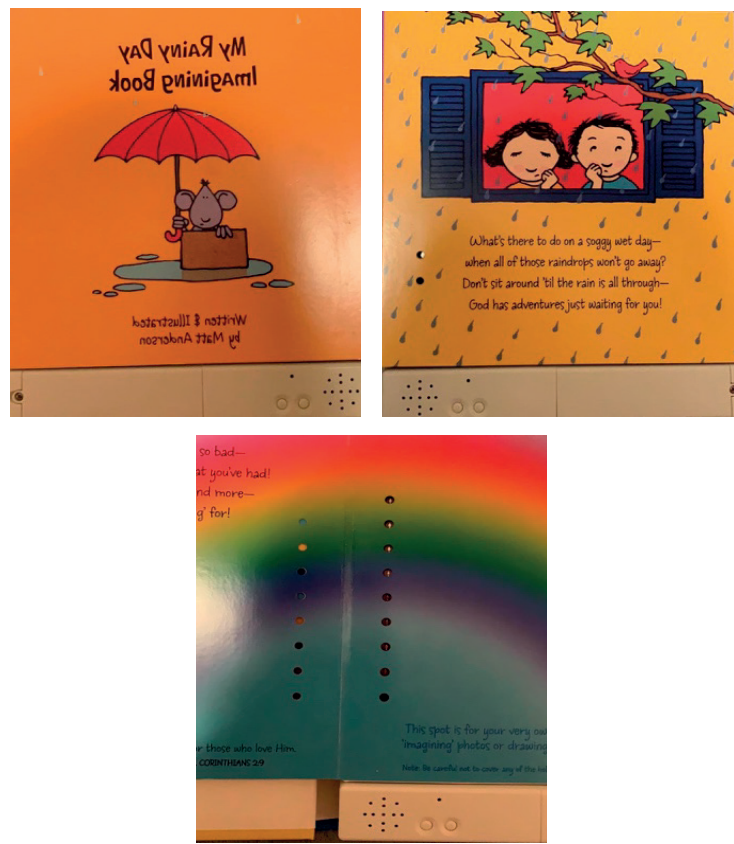

Este libro tiene audio en cada página, al momento de cambiar la hoja se apaga la canción, se abre y suena una nueva canción con referencia a cada escenario y sirve para el sentido del oído (Anderson, 2012).

\section{Bocetaje}

Para empezar el libro, se desarrollaron diferentes propuestas básicas.

\section{Figura 14 » Bocetos_parte}
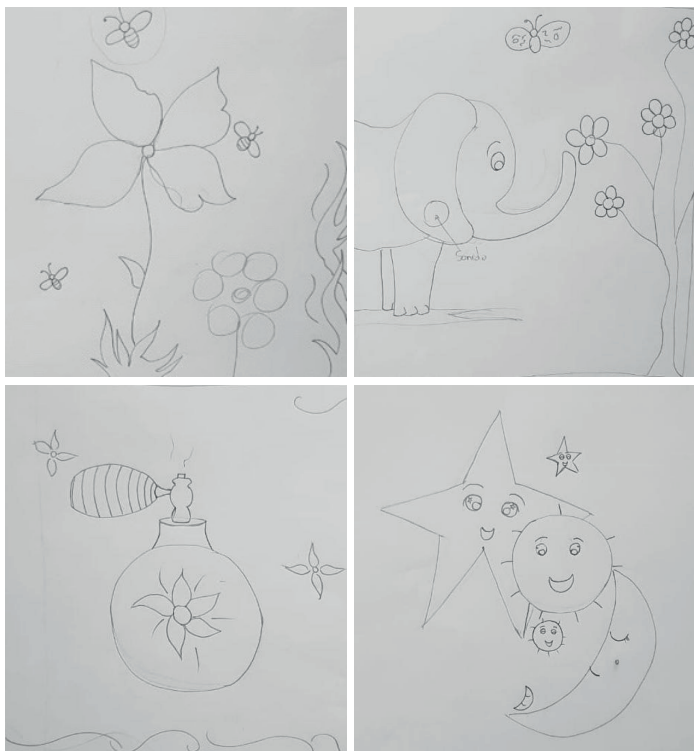

Bocetos aprobados, cada hoja tendrá su interactividad, se hicieron estas propuestas con la finalidad de que los niños puedan interactuar de manera sencilla, donde tengan la facilidad de reconocer los colores, las formas, donde aprecien la percepción de las texturas; plumas, arena, botones etc.

Figura 15 » Bocetos_parte2
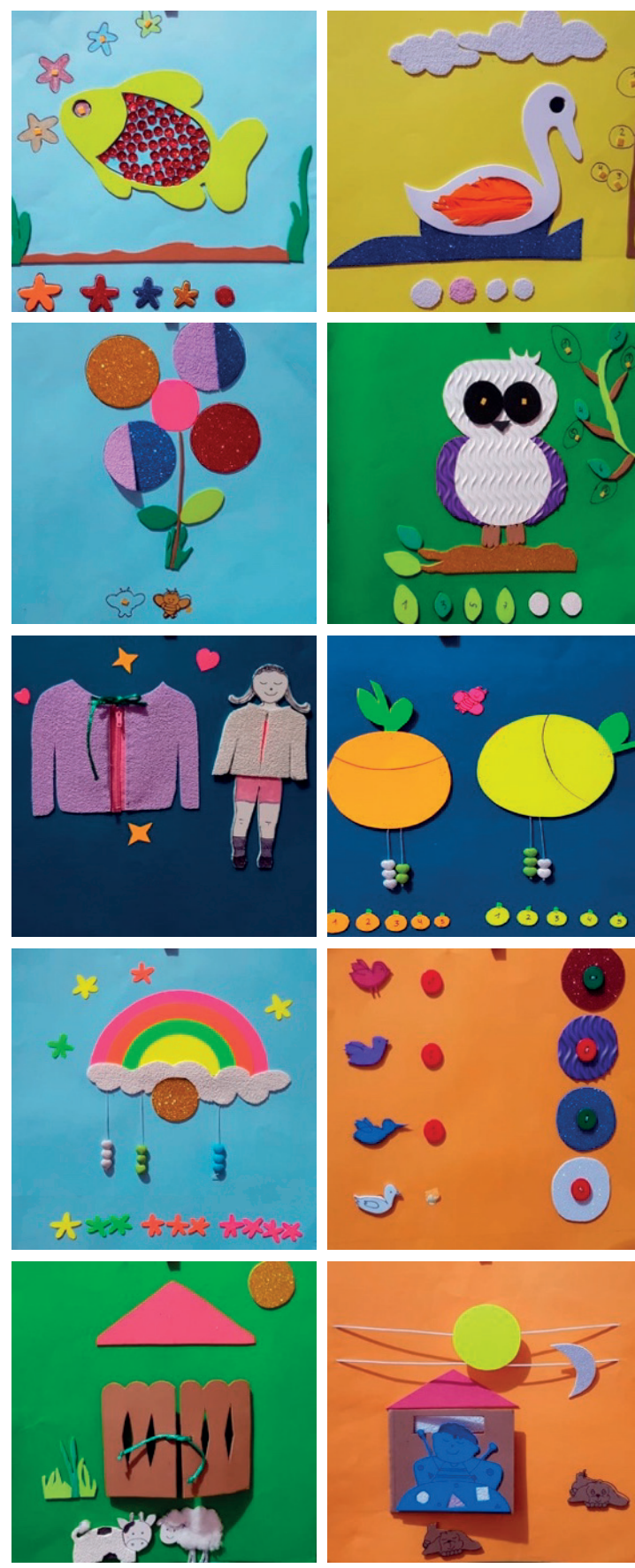


\section{Ilustración}

Se utilizó la herramienta Adobe Ilustrador para ya finiquitar las formas de cada página, juntamente con el fondo, para luego hacer un molde en fomix, cubierto de tela fieltro.

Figura 16 » Illustración_parte1
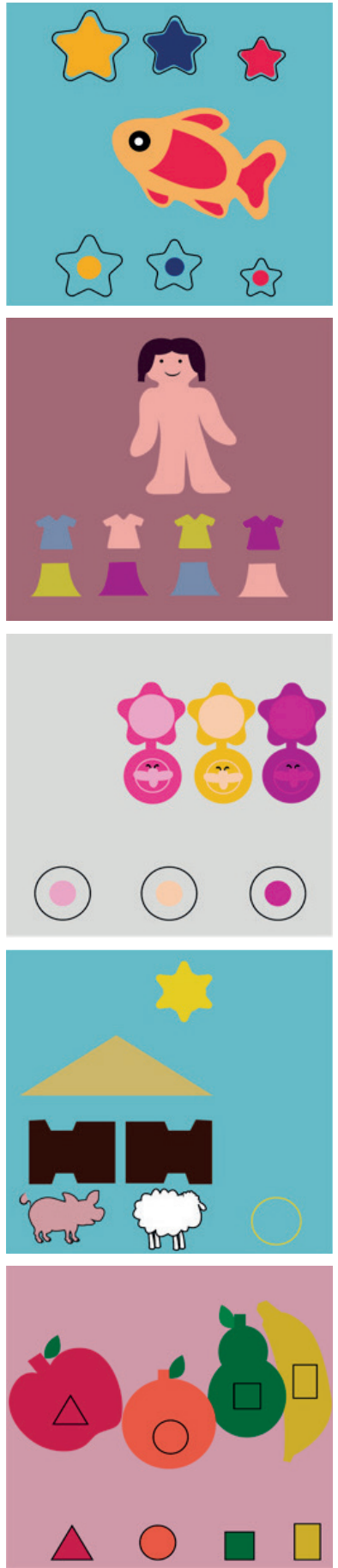
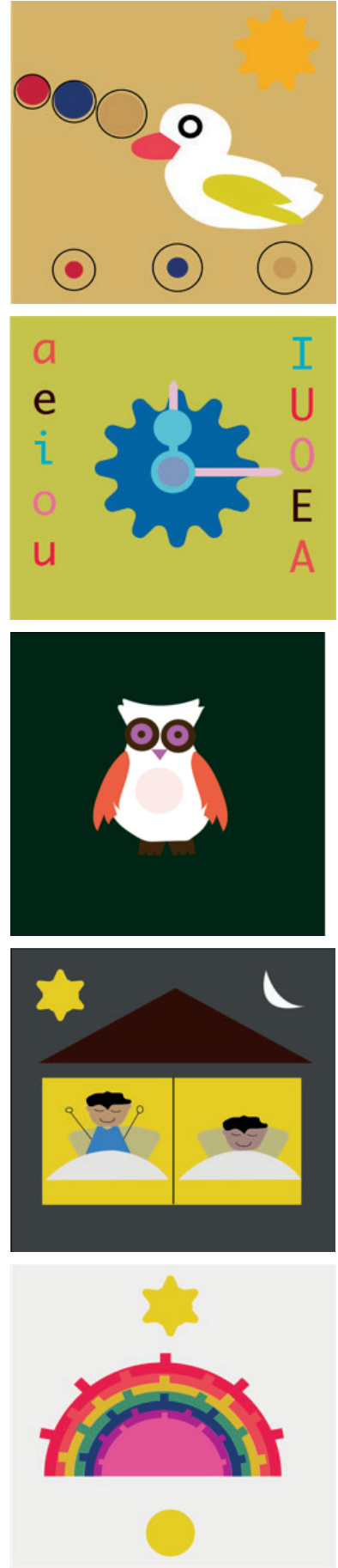

\section{Formato}

El formato depende de la comodidad de los niños, no debe ser pesado, es muy importante que el libro tenga espacio suficiente para que los niños puedan interactuar con cada figura y será con la ayuda de sus guías escolares. Su medida será 30x30 formato rígido en las cinco hojas, el fieltro tendrá una medida de 32x32 para coser los bordes.

\section{Aerografía y proceso}

Se utilizó aerografía con la finalidad de que las hojas se vean con más contraste y el niño pueda apreciar mejor las tonalidades en color.

\section{Figura 17 » Aereografía}

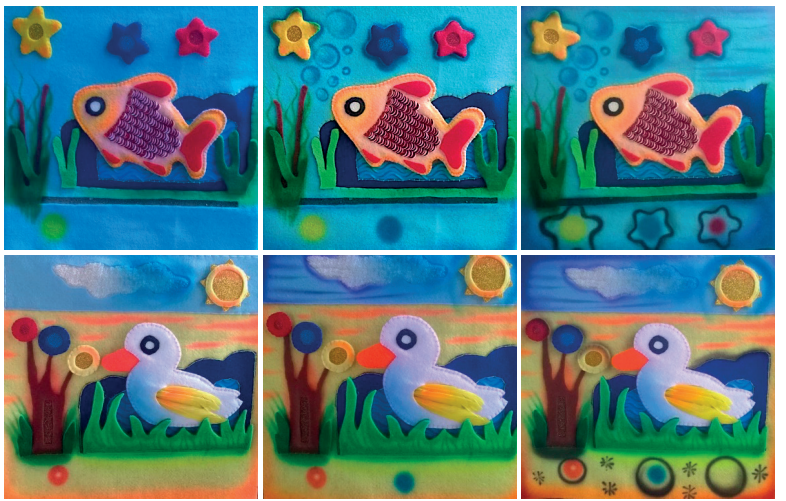

\section{Producto final "luces led"}

Las luces led, ayuda para el sentido auditivo y visual, en cuanto se abra cada página se tocará el pulsante correspondiente, empezará a sonar la música infantil acorde al ambiente y las luces empezarán a titilar para que los niños seleccionen cada figura y coloquen en el lugar que pertenece relacionado al color.

Figura 18 » Luces Led (Mariela Pauta)

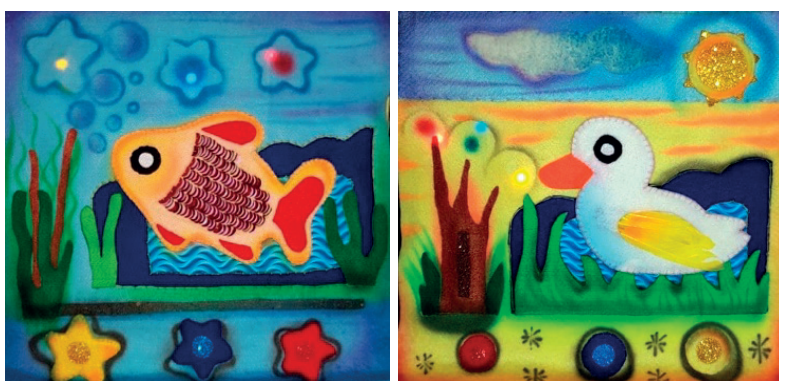




\section{RESULTADOS}

Se llevó a cabo un estudio de tipo cuasi-experimental en donde se aplicaron encuestas a docentes de educación especial y en el Centro de Estimulación Integral y Apoyo Psicoterapéutico, se realizaron actividades con 3 niños que poseen discapacidad intelectual. A continuación, se exponen las reacciones de los niños de acuerdo con el tipo de discapacidad que presentan:

\section{Discapacidad intelectual leve "Retraso en su desarrollo".}

1 Demostró interés en los juegos del libro, participó con normalidad, analizó las texturas.

2 Reaccionó a los audios.

3 Reconoció colores.

4 Relacionó formas.

\section{Discapacidad intelectual moderada "Síndrome de Down".}

1 Demostró interés los 2 primeros minutos.

2 Reconoció colores.

3 Relacionó formas.

4 Reaccionó a las texturas de las dos primeras páginas.

5 Fue muy hiperactivo y se enfocó más al audio.

\section{CONCLUSIONES}

El libro sensorial ayuda al desarrollo motriz de los niños con y sin discapacidad intelectual, debido a que despierta los cinco sentidos, generando la estimulación a temprana edad.

Los pequeños movimientos que realizan los niños "Motricidad Fina" ayudan a la coordinación "óculo-manual" que quiere decir coordinación entre los ojos y manos para agarrar una pintura y colorear, por ejemplo. Los libros sensoriales son de gran importancia porque es rico en estímulos. Además, un aspecto fundamental del libro es la inserción de luces led, lo cual permite que los niños se interesen más en trabajar y ejercer sus habilidades motrices, los audios de cada página permitirán una mayor dinámica y alegría para trabajar con el libro.

Uno de los principales factores para que los niños se diviertan aprendiendo, es utilizando el lenguaje visual, ya que la ilustración es un poderoso medio y recurso de comunicación en el área educativa.

\section{BIBLIOGRAFÍA}

Almanza, D. (s.f.). Desarrollo Sensorial. Obtenido de https://www.academia.edu: https://www.academia. edu/34886606/Desarrollo_sensorial

Anderson, M. (2012). My Rainy day imagining book. China.

APM. (2 de enero de 2018). Motricidad fina y gruesa. Obtenido de http://apoyopedagogicomanizales. blogspot.com: http://apoyopedagogicomanizales. blogspot.com/p/quienes-somos.html

Asociación Americana de Discapacidades Intelectuales y del Proceso . (2019). Obtenido de https://aaidd. org/intellectual-disability: https://aaidd.org/intellectual-disability/definition

Bassedas, E. (2010). Alumnado con discapacidad intelectual y retraso en el desarrollo. En E. Bassedas, Alumnado con discapacidad intelectual y retraso en el desarrollo (1." edición ed., Vol. VI, pág. 32). España, Barcelona.

CONADIS. (02 de Abril de 2019). Consejo Nacional para la igualdad de discapacidades. Recuperado el 02 de Abril de 2019, de https://www.consejodiscapacidades.gob.ec: https://www.consejodiscapacidades. gob.ec/estadisticas-de-discapacidad/

Educación Especial e Inclusión Educativa. UNESCO (2014)., Uruguay, Montevideo.

Educación Inclusiva. (2016). Obtenido de http://www. inclusioneducativa.org: www.inclusion-international.org

Guijarro, R. B. (2008). LA EDUCACIÓN INCLUSIVA: EL CAMINO HACIA EL FUTURO. Organización de las Naciones Unidas, para la educación, la ciencia y cultura.

Ley Orgánica de Discapacidades. (25 de SEPTIEMBRE de 2012). Obtenido de https://www.consejodiscapacidades.gob.ec: https://www.consejodiscapacidades. gob.ec/wp-content/uploads/downloads/2014/02/ ley_organica_discapacidades.pdf

Libros Sensoriales, método Montessori. (11 de Abril de 2016). De mi casa al mundo. Obtenido de https:// www.demicasaalmundo.com/blog/libros-sensoriales-de-inspiracion-montessori-con-sorteo/: https://www.demicasaalmundo.com 
Loos, S., \& Hoinkis, U. (2007). Las personas con discapacidad también juegan. (N. ediciones, Ed.) Madrid, España.

Dehasse, J. (1994). Desarrollo Sensorial , Emocional. Retrieved from http://www.voraus.com/adiestramientocanino/modules/wfsection/html/ a000449.pdf

López, B. (19 de Marzo de 2019). Discapacidad Intelectual, ¿Qué es?, disagnóstico, causas, tipos y consejos. Obtenido de https://blog.cognifit.com: https:// blog.cognifit.com/es/discapacidad-intelectual/

Miles, M. A. (2019). La inclusión.

Sánchez, C. F. (2014). Situaciones didácticas de psicomotricidad (Primera edición ed.). (E. Trillas, Ed.) México.

Suárez, J. M. (19 de septiembre de 2017). Infotecarios. Obtenido de Libros sensoriales que promueven el aprendizaje : $\mathrm{http}: / /$ www.infotecarios.com

UNESCO. (2014). ENSEÑANZA Y APRENDIZAJE, LOGRAR LA CALIDAD PARA TODOS (2da Edición ed.).Unicef . (2013). El desarrollo del niño en la primera infancia y la discapacidad. Obtenido de Unicef : https://www.unicef.org 